\title{
Optic Flow Computation from Cardiac MR Tagging Using a Multiscale Differential Method
} A Comparative Study with Velocity-Encoded MRI

\author{
A. Suinesiaputra ${ }^{1,2}$, L.M.J. Florack ${ }^{2}$, J.J.M. Westenberg ${ }^{1}$, \\ B.M. ter Haar Romeny ${ }^{2}$, J.H.C. Reiber ${ }^{1}$, and B.P.F Lelieveldt ${ }^{1}$ \\ 1 Division of Image Processing, Department of Radiology \\ Leiden University Medical Center, Leiden, the Netherlands \\ 2 Division of Biomedical Image Analysis, Department of Biomedical Engineering \\ Eindhoven University of Technology, Eindhoven, The Netherlands \\ a.suinesiaputra@lumc.nl
}

\begin{abstract}
The computation of an optic flow field to reconstruct a dense velocity field from a sequence of tagged MR images faces a major difficulty: a non-constant pixel intensity. In this paper, we resolved this problem by regarding the MRI sequence as density images, which adhere to a principle of conservation of intensity. Based on this principle, optic flow equations are developed based on Gaussian derivatives as differential operators. The multiscale optic flow method is applied to cardiac tagged MRI. A quantitative analysis is presented comparing the reconstructed dense velocity field with a directly acquired velocity field using the velocity-encoded (VEC) MRI.
\end{abstract}

\section{Introduction}

Motion analysis is becoming increasingly important in cardiovascular imaging. The cine-MR tagging protocol [1] enables the inspection of myocardial motion, because of temporary tag pattern in the myocardium wall. The tag pattern is induced within a tissue, which will follow the tissue deformation. The tissue motion is clearly visible through the deformed pattern.

Automatic reconstruction of a dense velocity field from tagged MRI is the next step toward a detailed cardiac motion analysis. The velocity field can be computed directly by following the apparent pixel movement, which can be derived using optic flow (OF) methods [2. A large number of different optic flow methods have been proposed (see [3] for a comparison between various OF methods). However, only a few are proposed for extracting the dense OF field from tagged MRI because of one major problem: the brightness variation problem.

In the OF computation, a constant pixel intensity is assumed. This is contained in the formulation that a total derivative of the image function $L$ is zero.

$$
\frac{d L}{d t}=0 \quad \text { or } \quad \nabla L \cdot \mathbf{v}=0 ; \mathbf{v} \in \mathbb{R}^{3}
$$


The MR signal however, linearly depends on the accumulated protons in a certain area. Therefore the tissue deformation causes variation in the pixel intensity due to the divergence of the flow. The contribution of this paper is twofold:

- we propose a new dense optic flow framework, that does not assume a constant pixel intensity, but a constant density. This greatly reduces the sensitivity to brightness variation over time, and therefore enables a more reliable reconstruction of a dense velocity field from tagging MR images, and

- we present the first direct comparison between reconstructed and directly acquired (using VEC MRI) dense velocity field in clinically representative cases.

The remainder of this paper is structured as follows. Section 2 discusses our approach to the brightness variation problem in tagging MRI and the OF method in detail. In Section 3 , results from applying the optic flow method to cardiac MR tagging sequences are presented, and statistically compared to the corresponding velocity-encoded (VEC) MR images. Section 4 concludes with a discussion.

\section{Methodology}

\subsection{Conservation Principle in Tagging MRI}

Let $L: \mathbb{R}^{3} \rightarrow \mathbb{R}$ be a raw image function and $\mathcal{L}_{\mathbf{v}}$ be the Lie derivative, a generalization notion of the directional derivative of a function, with respect to a spatiotemporal vector $\mathbf{v} \in \mathbb{R}^{3}$. The optic flow field is defined as a spatiotemporal vector field that satisfies the following constraint

$$
\mathcal{L}_{\mathbf{v}} L=0
$$

This is called the Optic Flow Constraint Equation (OFCE). It defines the mathematical concept of the optic flow field in a more general formulation than Eq. [1]

Florack et. al. defined two different kind of pixel flows in an image: scalar and density images [4. In scalar images, the pixel intensity is assumed to be constant. Horn \& Schunck's OF equation [2] deals with these images. In density images, the conserved quantity is not a single pixel value, but the intensity is integrated over a local region. Pixel intensities in the density images may vary, but its total integral is conserved: the local "intensity mass" is preserved.

Tagging MRI is a typical example of density images, because the MR signal is formed by the net magnetization of excited protons [5]. The total number of protons in a tissue is preserved, even if the tissue is deformed. Optic flow analysis of tagging MRI therefore greatly benefits from the derivation of OFCE based on the density conservation principle.

Let $L_{\rho}: \mathbb{R}^{3} \rightarrow \mathbb{R}$ be a raw image function that holds the density images property. The Lie derivative of $L_{\rho}$ with respect to a vector $\mathbf{v}$ is defined by taking the derivative of the density function together with the vector field.

$$
\mathcal{L}_{\mathbf{v}} L_{\rho}=\sum_{\mu=1}^{3} \partial_{\mu}\left(L_{\rho} \mathbf{v}^{\mu}\right)=\nabla \cdot\left(L_{\rho} \mathbf{v}\right)=0
$$


Equation 3 is the OFCE definition for the density images. It has an interesting physical interpretation as the divergence of a vector $\mathbf{v}$ representing the rate of expansion per unit volume under the flow. Thus it accounts for the change of volume of the local integrated region. In the next sections, unless stated otherwise, we use the notation of $L$ for the density images.

\subsection{The First Order Density Multiscale OFCE}

Let $\mathbf{v}^{T}=(w(\mathbf{x}), u(\mathbf{x}), v(\mathbf{x})), \mathbf{x} \in \mathbb{R}^{3}$ be the optic flow vector, which equals the spatiotemporal vector $\mathbf{v}$ in Eq. 2 but in a more general form. The function $w: \mathbb{R}^{3} \rightarrow \mathbb{R}_{+}$is the temporal component and $u, v: \mathbb{R}^{3} \rightarrow \mathbb{R}$ are the spatial components in $x$ and $y$ directions respectively.

As the Lie derivative vanishes in Eq. 3, its convolution with a Gaussian kernel in the scale-space framework also vanishes. Therefore Eq. 3 in the Gaussian scalespace framework will be

$$
-\int_{\mathbf{R}^{3}} L\left(\nabla \phi_{\sigma, \tau} \cdot \mathbf{v}\right) d \mathbf{x}=0, \quad \sigma, \tau \in \mathbb{R}_{+}
$$

where $\phi_{\sigma, \tau}$ is a three dimensional Gaussian kernel with an isotrophic spatial scale $\sigma$ and a temporal scale $\tau$.

To eliminate the aperture problem [6], an additional constraint is required. Since the exact cardiac motion is not a-priori known, we used a vector that is perpendicular to the tangential vector as the additional constraint: the normal flow constraint. If $\mathbf{v}^{T}=(w, u, v)$ is the normal vector, then $\mathbf{v}_{t}^{T}=(0,-v, u)$ is its tangential vector. We can substitute $\mathbf{v}$ and $\mathbf{v}_{t}$ into Eq. 4 to get a unique solution.

Another constraint is the temporal gauge constraint, which means fixing $w(\mathbf{x}) \rightarrow 1$. This states that there are no creation or elimination of pixel intensities. Using Eq. 4 for the normal and tangential vector and imposing the temporal gauge condition, we can define the first order OFCE for the density image. It consists of 8 unknowns (the two components of flow vectors and their derivatives with respect to $x, y$ and $t$ ) in 8 linear equations:

$$
\begin{aligned}
-L_{t}= & L_{x} u+L_{y} v+\tau^{2} L_{x t} u_{t}+\tau^{2} L_{y t} v_{t}+\left(L+\sigma^{2} L_{x x}\right) u_{x}+\sigma^{2} L_{x y} v_{x}+ \\
& \sigma^{2} L_{x y} u_{y}+\left(L+\sigma^{2} L_{y y}\right) v_{y} \\
-L_{t t}= & L_{x t} u+L_{y t} v+\left(L_{x}+\tau^{2} L_{x t t}\right) u_{t}+\left(L_{y}+\tau^{2} L_{y t t}\right) v_{t}+\left(L_{t}+\sigma^{2} L_{x x t}\right) u_{x}+ \\
& \sigma^{2} L_{x y t} v_{x}+\sigma^{2} L_{x y t} u_{y}+\left(L_{t}+\sigma^{2} L_{y y t}\right) v_{y} \\
-L_{x t}= & L_{x x} u+L_{x y} v+\tau^{2} L_{x x t} u_{t}+\tau^{2} L_{x y t} v_{t}+\left(2 L_{x}+\sigma^{2} L_{x x x}\right) u_{x}+ \\
& \left(L_{y}+\sigma^{2} L_{x x y}\right) v_{x}+\sigma^{2} L_{x x y} u_{y}+\left(L_{x}+\sigma^{2} L_{x y y}\right) v_{y} \\
-L_{y t}= & L_{x y} u+L_{y y} v+\tau^{2} L_{x y t} u_{t}+\tau^{2} L_{y y t} v_{t}+\left(L_{y}+\sigma^{2} L_{x x y}\right) u_{x}+\sigma^{2} L_{x y y} v_{x}+ \\
& \left(L_{x}+\sigma^{2} L_{x y y}\right) u_{y}+\left(2 L_{y}+\sigma^{2} L_{y y y}\right) v_{y} \\
0= & -L_{y} u+L_{x} v-\tau^{2} L_{y t} u_{t}+\tau^{2} L_{x t} v_{t}-\sigma^{2} L_{x y} u_{x}+\left(L+\sigma^{2} L_{x x}\right) v_{x}- \\
& \left(L+\sigma^{2} L_{y y}\right) u_{y}+\sigma^{2} L_{x y} v_{y} \\
0= & L_{y t} u-L_{x t} v+\left(L_{y}+\tau^{2} L_{y t t}\right) u_{t}-\left(L_{x}+\tau^{2} L_{x t t}\right) v_{t}+\sigma^{2} L_{x y t} u_{x}- \\
& \left(L_{t}+\sigma^{2} L_{x x t}\right) v_{x}+\left(L_{t}+\sigma^{2} L_{y y t}\right) u_{y}-\sigma^{2} L_{x y t} v_{y} \\
0= & L_{x y} u-L_{x x} v+\tau^{2} L_{x y t} u_{t}-\tau^{2} L_{x x t} v_{t}+\left(L_{y}+\sigma^{2} L_{x x y}\right) u_{x}- \\
& \left(2 L_{x}+\sigma^{2} L_{x x x}\right) v_{x}+\left(L_{x}+\sigma^{2} L_{x x y}\right) u_{y}-\sigma^{2} L_{x x y} v_{y} \\
0 & L_{y y} u-L_{x y} v+\tau^{2} L_{y y t} u_{t}-\tau^{2} L_{x y t} v_{t}+\sigma^{2} L_{x y y} u_{x}-\left(L_{y}+\sigma^{2} L_{x x y}\right) v_{x}+ \\
& \left(2 L_{y}+\sigma^{2} L_{y y y}\right) u_{y}-\left(L_{x}+\sigma^{2} L_{x y y}\right) v_{y}
\end{aligned}
$$


where $L_{\mu}$ is the image derivative in the Gaussian scale space representation, defined as the convolution of the original image $L$ (as the initial condition) with the Gaussian derivative kernel $\phi_{\sigma, \tau}$ in the $\mu$ dimension [7]. Although there are derivatives of each velocity component in Eq. 5. we only present here the $u$ and $v$ component, as $x$ and $y$ velocity component respectively.

\subsection{The Multiscale Scheme}

There is still one parameter left unexplored in Eq. 5 the scale parameters $\sigma$ and $\tau$. The scale is a free parameter, however one proper scale is enough to get a unique solution. Niessen et. al. [8] has studied a scale selection method based on a numerical stability of the solution. The "best result" is defined numerically as the most stable solution of the linear equation system in Eq. 囵. We applied the same method, using the Frobenius norm of the coefficient matrix of Eq. 5 as the stability measurement.

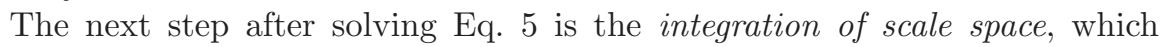
smoothes the output optic flow field [9]. We modified the energy minimization in 9] into the convolution with Gaussian kernels.

Let $\tilde{\mathbf{v}}(\mathbf{x}), \mathbf{x} \in \mathbb{R}^{3}$ be an optic flow vector after the integration and $\mathbf{v}_{\sigma, \tau}(\mathbf{x})$ be an optic flow vector after the computation of Eq. 5 with spatial scale $\sigma$ and temporal scale $\tau$. The notion of $\sigma, \tau$ in the vector $\mathbf{v}$ is added to incorporate the scale selection scheme. The integration of scale space is given by the following convolution process:

$$
\tilde{v}^{\mu}\left(\mathbf{x}_{\mathbf{0}}\right)=\int_{\mathbf{x} \in \mathbb{R}^{3}} p\left(\mathbf{x}_{\mathbf{0}}\right) v_{\sigma, \tau}^{\mu}\left(\mathbf{x}_{\mathbf{0}}\right) \phi_{\sigma, \tau}\left(\mathbf{x}-\mathbf{x}_{\mathbf{0}}\right) d \mathbf{x}
$$

where $\mu$ is one of vector's components, $p(\mathbf{x})$ is a penalty function and $\phi_{\sigma, \tau}(\mathbf{x})$ is the Gaussian kernel. The penalty function $p(\mathbf{x})$ in Eq. [6] is defined as:

$$
p(\mathbf{x})=\exp \left(-\frac{\lambda \kappa(\mathbf{x})}{N_{\kappa}}\right)
$$

where $\kappa(\mathbf{x})$ is the Frobenius norm of the coefficient matrix of Eq. 5 at the spatiotemporal position $\mathbf{x}$. The value $\lambda$ is a constant in the range of $(0 . .1]$ and $N_{\kappa}$ is a normalization factor. The value of Eq. 7 decreases exponentially when $\kappa$ is large, which means that the more unstable solution contributes less in the final optic flow result.

\section{Experimental Results}

The multiscale OF method for tagged MRI has been tested and validated on several analytical images and tagging MRI of a phantom agar [10. In this paper, the method is applied to real cardiac tagged MR images from a number of cardiac-healthy subjects. The OF method is restricted to estimate only for in-planar motion (2D) in tagging MRI. The results are compared to directly acquired VEC MRI. 


\subsection{Clinical Data}

Eight healthy volunteers were selected without history of valvular disease, proven from echocardiography. VEC MRI images were acquired in a short-axis orientation at a mid-ventricular level. A standard spoiled gradient-echo was applied with velocity-encoding in three directions (maximal velocity sensitivity is 20 $\mathrm{cm} / \mathrm{s}$ ). Retrospective gating with delayed reconstruction was used to cover the full cardiac cycle (30 phases). This acquisition was performed during free breathing.

An MR tissue tagging sequence is used in the same short-axis orientation and position for comparison. Rectangular grid tagging is performed with tag grid spacing $=8.3 \mathrm{~mm}$. Prospective triggering is used with maximum number of heart phases reconstructed, resulting in typical 20-30 phases during one cardiac cycle. This acquisition is performed under breath-holding (in expiration).

Both the VEC and tagging MR images for all patients were acquired in the same study time, with same patient positioning. Due to different breathing conditions, the left ventricular (LV) contours were drawn separately. Contours for tagging images were drawn manually using a dedicated cardiac MR analytical software package (MRI-MASS [1]). Contours were drawn in the regular shortaxis image, at the closest slice position to the tagging image, because of the better visibility of the myocardial contours in (non-tagged) short-axis slices. Contours for the VEC MRI were drawn manually in the through-plane velocity image, because it gives clearer definition of the myocardial wall than the in-plane velocity images.

In the analysis, time phases of tagging and VEC sequences were normalized into a single cycle. Since the number of phases in tagging images was not equal, we interpolated 30 time frames in tagging according to the time steps in the VEC MRI.

\subsection{Results}

The region of interest is the LV myocardium. Figure 1 shows one sample result of the OF field from a subject compared visually with their corresponding VEC MR images at mid-systole and mid-diastole phases. Only vectors inside the LV myocardium are shown. Notice how the tagging patterns are fading at later phases.

For this comparison between VEC MRI and the computed OF from tagging, we only looked at the in-plane motion of the VEC MRI. Therefore the z-velocity components were discarded. We also looked at the global LV wall motion, instead of regional wall motion, because the scope of this paper is to investigate how the LV wall motion from optic flow globally relates to VEC MRI.

The LV wall undergoes two basic motions, i.e. radial and circumferential components. The radial component defines contraction motion relative to the center of the LV, while the circumferential defines the torsion movement. Figure2 shows the comparison of the mean global radial and circumferential velocity components between the computed OF and VEC MRI. 


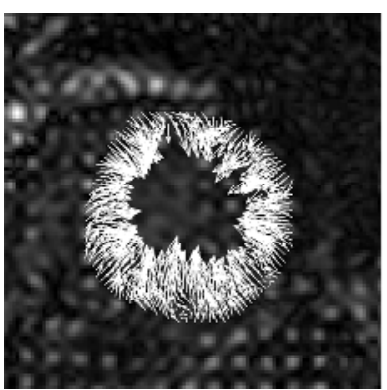

(a) Mid-systole dense OF

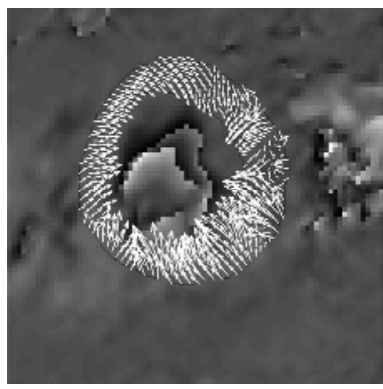

(a) Mid-systole VEC

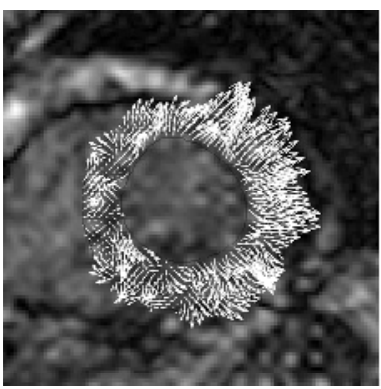

(b) Mid-diastole dense $\mathrm{OF}$

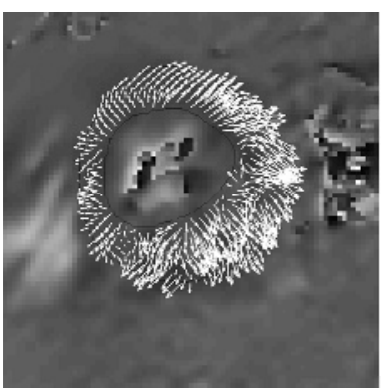

(b) Mid-diastole VEC

Fig. 1. One sample comparison result between dense OF from tagging and VEC MRI.

Table 1. Correlation coefficients

\begin{tabular}{|l|c|c|c|}
\hline Components & Full cycle & Systolic & Diastolic \\
\hline Radial & $r=0.86(S D=0.04)$ & $r=0.98(S D=0.01)$ & $r=0.71(S D=0.13)$ \\
\hline Circumferential & $r=0.42(S D=0.17)$ & $r=0.52(S D=0.18)$ & $r=0.23(S D=0.29)$ \\
\hline
\end{tabular}

We calculated the correlation coefficient for each components to investigate the relation between the OF and VEC MRI. As can be seen in Fig. 3, the OF and the VEC radial velocity has high correlation $(r=0.86)$. This is not the case for the circumferential velocity $(r=0.42)$. Also the radial velocity correlates better at the systolic part of the cycle, while the diastolic half (second half cycle) is less correlated (Tab. 1). In the scatter plot (Fig. 3), this phenomenon is shown by a cluster of systolic plots (asterisk signs) and diastolic plots (plus signs).

\section{Discussion}

The circumferential component correlates less good $(r=0.42)$. This can be explained by two factors. The circumferential movements in the VEC images are more visually apparent than the circumferential movements in the tagging images. This may be caused by the longer trigger delay time of the tagging images, i.e. the rapid torsion at the start of the contraction is not sufficiently 

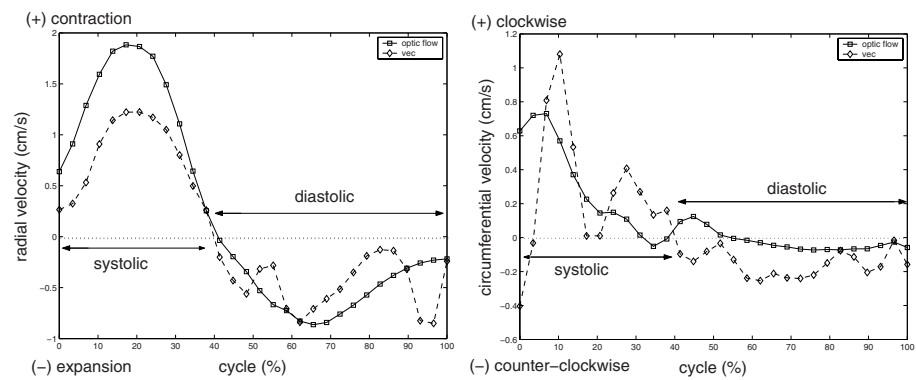

Fig. 2. Mean of global radial (left) and circumferential (right) components from 8 subjects.

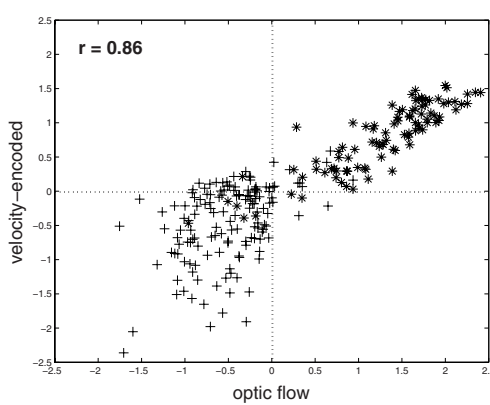

(a) Radial component

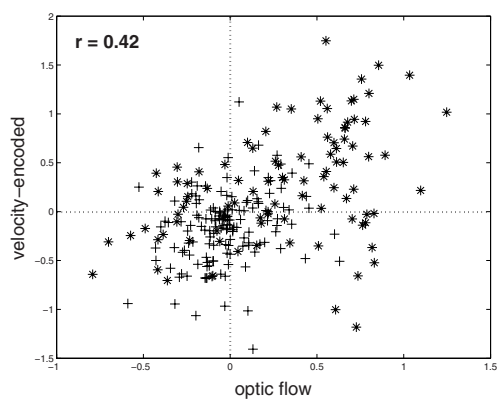

(b) Circumferential component

Fig. 3. Scatter plot of radial (left) and circumferential (right) component. Asterik ('*') signs are the systolic part, while plus ('+') signs are the diastolic part.

covered by the tagging sequence. Therefore we cannot expect the optic flow method to produce the motion that is not sufficiently present in the image data.

Moreover the aperture problem was solved using the normal flow constraint, which reduces all pixel motions to be in the direction of the image gradient. In order to overcome this limitation, the normal flow constraint should be replaced by a more knowledge-driven motion constraint. If we integrate a-priori knowledge of the LV wall motion, for instance taking into account the torsion movement, then it would be better to replace the normal flow constraint with this knowledge.

The computed dense OF field from tagged MRI shows a very good correlation with the VEC MRI for the LV wall radial contraction. Especially in the systolic part of the cardiac cycle this correlation is stronger $(r=0.98$ in systole and $r=0.71$ in diastole). In most cases, the cardiac systolic function is clinically more meaningful than the diastolic part. Systolic function gives information of how well the heart can pump the blood to the whole body. From this, we conclude that the proposed dense OF method shows a promising non-invasive technique to assess the velocity field during the systolic part of the cardiac cycle.

The proposed method has the flexibility to be extended to $4 \mathrm{D}$ by adding one more spatial component in the spatiotemporal vector definiton. The optic flow 
equations would become more complex and more additional constraint equations are needed. This is the topic of ongoing research.

\section{References}

[1] Axel, L., Dougherty, L.: MR Imaging of Motion with Spatial Modultion of Magnetization. Radiology 171 (1989) 841-845

[2] Horn, B.K.P., Schunck, B.G.: Determining Optical Flow. Artificial Intelligence 17 (1981) 185-203

[3] Beauchemin, S.S., Baron, J.L.: The Computation of Optical Flow. ACM Computing Surveys 27 (1995) 433-467

[4] Florack, L., Niessen, W., Nielsen, M.: The Intrinsic Structure of Optic Flow Incorporating Measurement Duality. International Journal of Computer Vision 27 (1998) 263-286

[5] Nitz, W., Reimer, P.: Contrast Mechanism in MR Imaging. European Radiology 9 (1999) 1032-1046

[6] Marr, D.: Vision. W.H. Freeman \& Co., San Fransisco (1982)

[7] Lindeberg, T.: Scale Space Theory in Computer Vision. Kluwer Academic Publishers, Dordrecht (1994)

[8] Niessen, W., Mass, R.: Optic Flow and Stereo. In Sporring, J., ed.: Gaussian Scale-Space Theory. Kluwer (1997) 31-42

[9] Pedersen, K.S., Nielsen, M.: Computing Optic Flow by Scale-Space Integration of Normal Flow. In Kerckhove, M., ed.: Scale-Space 2001. Number 2106 in Lecture Notes in Computer Science. Springer-Verlag (2001) 14-25

[10] Suinesiaputra, A.: Multiscale Optic Flow Analysis For Magnetic Resonance Tagging. BMT-Report 2002-02, Department of Biomedical Engineering, Technische Universiteit Eindhoven (2002)

[11] van der Geest, R.J., Jansen, E., Reiber, J.H.C.: Automated Detection of Left Ventricular Epi- and Endocardial Contours in Short-Axis MR Images. Computers in Cardiology (1994) 245-248 